\title{
Add-on anticholinergic therapy for residual nocturia in patients with lower urinary tract symptoms receiving $\alpha 1$-blocker treatment: a multi-centre, prospective, randomised study
}

\author{
Osamu Yokoyama • Akira Tsujimura • Hironobu Akino • Naoki Segawa · Satoshi Tamada • Naoki Oguchi · \\ Yasuhide Kitagawa · Hidenori Tsuji · Akihiko Watanabe $\cdot$ Teruo Inamoto $\cdot$ Nobutaka Shimizu $\cdot$ Yasuyoshi Fujiuchi \\ Yoji Katsuoka · Haruhito Azuma · Tadashi Matsuda $\cdot$ Mikio Namiki $\cdot$ Hirotsugu Uemura $\cdot$ Akihiko Okuyama \\ Norio Nonomura · Hideki Fuse · Tatsuya Nakatani
}

Received: 2 May 2014 / Accepted: 1 September 2014 / Published online: 16 September 2014

(C) The Author(s) 2014. This article is published with open access at Springerlink.com

\begin{abstract}
Purpose To evaluate the efficacy and safety of imidafenacin (IM), a novel short half-life anticholinergic, as addon therapy for male LUTS with nocturia and nocturnal polyuria.

Materials and methods This multicenter, prospective, randomized, open-labelled study was conducted and involved men who had frequency, urgency, and nocturia despite receiving a stable dose of $\alpha 1$-blocker for $\geq 1$ month. Subjects were randomised to control ( $\alpha 1$-blocker alone), IM twice/day ( $\alpha 1$-blocker $+0.1 \mathrm{mg}$ imidafenacin twice daily), or IM nightly ( $\alpha 1$-blocker plus $0.1 \mathrm{mg}$ imidafenacin nightly) group; the treatment period was 8 weeks. Primary endpoints included improvements in night-time frequency and Nocturia Quality of Life Questionnaire (N-QOL) scores. Secondary endpoints included changes from the
\end{abstract}

Trial identification: The clinical study design is posted at http://www.umin.ac.jp/ctr/ (Unique ID UMIN000002344).

O. Yokoyama $(\bowtie) \cdot$ H. Akino

Department of Urology, Faculty of Medical Science, University of Fukui, Fukui, Japan

e-mail: oyoko@u-fukui.ac.jp

A. Tsujimura $\cdot$ A. Okuyama $\cdot$ N. Nonomura

Department of Urology, Osaka University Graduate School

of Medicine, Suita, Japan

N. Segawa $\cdot$ T. Inamoto $\cdot$ Y. Katsuoka $\cdot$ H. Azuma

Department of Urology, Osaka Medical College, Osaka, Japan

S. Tamada $\cdot$ T. Nakatani

Department of Urology, Osaka City University, Osaka, Japan

N. Oguchi $\cdot$ T. Matsuda

Department of Urology and Andrology, Kansai Medical

University, Osaka, Japan baseline in frequency volume chart variables, and post-void residual volume.

Results and limitations Compared with the controls, IM twice/day and IM nightly patients had a significantly lower night-time frequency (changes from baseline: $0.1 \pm 0.8$ in control, $-0.6 \pm 0.9$ in IM twice/day, and $-0.4 \pm 1.0$ in IM nightly, $p=0.5227,0.0006$ and 0.0143 , respectively). The hours of undisturbed sleep and N-QOL score were significantly improved in IM twice/day group, though not IM nightly group. Nocturnal urine volume was significantly reduced in IM nightly group, although total urine volume remained unchanged.

Conclusions A short half-life anticholinergic is suggested to be safe and effective as an add-on therapy for residual nocturia in patients with male LUTS receiving $\alpha 1$-blocker treatment. Anticholinergic administration nightly could reduce the nocturnal urine volume.

Y. Kitagawa $\cdot$ M. Namiki

Department of Integrative Cancer Therapy and Urology,

Kanazawa University Graduate School of Medical Science,

Kanazawa, Japan

H. Tsuji $\cdot$ N. Shimizu $\cdot$ H. Uemura

Department of Urology, Kinki University School of Medicine,

Osaka, Japan

A. Watanabe $\cdot$ Y. Fujiuchi $\cdot$ H. Fuse

Department of Urology, Graduate School of Medicine

and Pharmaceutical Sciences for Research, University of Toyama,

Toyama, Japan 
Keywords Anticholinergic $\cdot$ Nocturia $\cdot$ Nocturnal urine volume $\cdot$ Overactive bladder $\cdot$ Benign prostatic hyperplasia $\cdot$ Imidafenacin

\section{Introduction}

Nocturia (night-time voiding) — defined as 'the need to get up once or more times for nocturnal voids' [1] —is the most common lower urinary tract symptom (LUTS), reportedly observed in up to $69 \%$ of cases [2]. Since its prevalence increases with age [2,3], it is more common in the elderly, and it greatly affects general health and quality of life [4-6]. Many individuals with nocturia, particularly elderly men, have other LUTS, including urinary frequency, weak stream, and urgency. In men, these symptoms are primarily attributed to benign prostatic hyperplasia (BPH). Therapy typically involves treatment for detrusor overactivity or bladder outlet obstruction. However, while either or both these aetiologies may underlie nocturia, therapy may fail because of an often overlooked component, namely nocturnal polyuria (NP) [7].

Treatment for voiding and/or storage symptoms suggestive of BPH is now the initial choice for nocturia, and $\alpha_{1^{-}}$ blockers, which target the dynamic component of prostatic obstruction, remain the most widely used pharmacological agents [8]. $\alpha_{1}$-Blockers for LUTS suggestive of BPH are expected to alleviate storage symptoms including nocturia. However, they are not adequately effective for nocturiarelated sleep disturbance. Anticholinergics with $\alpha_{1}$-blockers have shown statistical success in some groups, but the clinical significance of their effects is limited [9]. Anticholinergics reportedly improve overactive bladder (OAB) in nocturia by increasing bladder capacity but have no effect on NP [10]. We previously reported that imidafenacin decreased urine volume via suppression of C-fibres in the rat bladder [11] and clinically improved nocturia and reduced nocturnal urine volume [12]. However, the latter study was conducted mainly for female OAB patients. Thus, it remains unclear whether anticholinergics are effective for treating nocturia and NP, especially among men. Studies have reported that anticholinergics with $\alpha_{1}$-blockers are effective and safe for male LUTS [13-15]. However, these studies did not focus on nocturia and NP evaluated using urinary frequency volume charts (FVCs). Only limited data support the use of $\alpha_{1}$-blocker and anticholinergics; moreover, nocturia has rarely been used as a primary endpoint when studying these drug classes, and such studies have not consistently controlled for the effect of NP [16].

In this study, we investigated whether imidafenacin, a novel anticholinergic that has been marketed in Japan since $2007[17,18]$, is effective for treating nocturia and NP.

\section{Materials and methods}

This multi-centre, randomised, 8-week study was conducted at eight university hospitals in Japan between August 2009 and March 2011. The study, named the GoodNight study, complied with the International Conference on Harmonization Good Clinical Practice Guideline and the Declaration of Helsinki. Further, the study protocol was approved by the review board of each hospital, and all subjects provided written informed consent. The clinical study design is posted at http://www.umin.ac.jp/ctr/ (Unique ID: UMIN000002344).

\section{Subject selection}

Before the subjects were enrolled in this study, they underwent behavioural therapy, including lifestyle guidance. Eligible subjects included men with persistent nocturia $(\geq 2$ voids/night) and LUTS including OAB symptoms [mean urinary frequency $\geq 8$ times $/ 24 \mathrm{~h}$ and $\geq 1$ micturitionrelated urgency episode/week, evaluated using the Overactive Bladder Symptom Score (OABSS) [19]] who had been receiving a stable dose of an $\alpha_{1}$-blocker for $\geq 1$ month. The International Prostate Symptom Score (IPSS)/IPSS-QOL was also evaluated. The exclusion criteria were as follows: post-void residual volume (PVR) $\geq 50 \mathrm{~mL}$, complications that are contraindications for anticholinergics, high possibility of prostate and bladder cancer, acute active urinary tract infection, indwelling or intermittent urethral catheterisation, comorbidities that affect nocturia (sleep apnoea syndrome, restless leg syndrome, insomnia, etc.), hormone or 5- $\alpha$-reductase inhibitor therapy that was started within the past 6 month, shift work and circadian rhythm disorder, irregular lifestyle, electrostimulation therapy or bladder training in the 10 days before the run-in period, contraindication for imidafenacin (primary angle-closure glaucoma, urinary retention, obstructive intestinal disease, paralytic ileus, gastrointestinal atony, and myasthenia gravis), and ineligibility as judged by the investigator in charge.

\section{Study design}

Eligible subjects were randomised to receive an 8-week continuous treatment with an $\alpha_{1}$-blocker alone (control), $\alpha_{1}$-blocker $+0.1 \mathrm{mg}$ imidafenacin twice daily (IM twice/ day), or $\alpha_{1}$-blocker plus $0.1 \mathrm{mg}$ imidafenacin nightly (IM nightly). The subjects were randomly assigned to the three groups in a random sequence. Random assignment was performed by the central registration system of an independent organisation, and age was used as a factor in the assignment. The $\alpha_{1}$-blockers were selected from common ones used in Japan (tamsulosin, naftopidil, and silodosin). 


\section{Clinical assessments}

The primary efficacy endpoints were improvements in night-time frequency as determined by the FVC, which was a 3-day bladder diary, and the Nocturia Quality of Life Questionnaire (N-QOL) score [20]. The N-QOL includes two domains, namely Sleep/Energy and Bother/Concern, and the total score and domain scores were calculated on a scale of 0-100, with 100 indicating ideal conditions. Secondary endpoints were other FVC variables, including hours of undisturbed sleep (HUS), 24-h micturition frequency, daytime micturition frequency, nocturnal/daytime urine volume, nocturnal polyuria index (NPi; defined as nocturnal urine volume/24-h urine output), urine volume voided/void, PVR, OABSS, IPSS, IPSS-QOL, and at baseline and at weeks 4 and 8. NP was diagnosed if the NPi was $\geq 33 \%$. Prostate volume and PVR were measured by ultrasound.

Adverse events (AEs) were classified by the investigator according to severity and relationship to the treatment.

\section{Statistical analysis}

Differences in FVC variables between treatment groups at baseline were evaluated using analysis of variance (ANOVA) at a significance level of $5 \%$; the N-QOL score and OABSS were evaluated using the two-sided KruskalWallis test. Intra- and intergroup numerical changes from baseline to 4 and 8 weeks were evaluated using paired and unpaired $t$ tests, respectively (control vs. IM twice/day or IM nightly). Intra- and intergroup changes in categorical variables, including the N-QOL score and OABSS, from baseline to 4 and 8 weeks were evaluated using the Wilcoxon and Mann-Whitney $U$ tests, respectively (control vs. IM twice/day or IM nightly). The distribution of $\alpha_{1}$-blocker use during treatment and the mean duration of $\alpha_{1}$-blocker use before study entry were evaluated using Fisher's exact test. Assessments of safety and tolerability were based on the findings for all patients who received at least one dose of the study medication. A two-sided significance level of $5 \%$ was applied for all statistical tests, except those conducted on baseline characteristics. All analyses were performed using SAS 9.1 (SAS Institute Inc., Cary, NC, USA).

\section{Results}

Subject population

Patient disposition is summarised in Fig. 1. The baseline demographic and clinical characteristics were similar among the three treatment groups (Table 1). From the FVCs, $78 \%(101 / 130)$ of the subjects were found to have
NP. The distribution of $\alpha_{1}$-blocker use did not differ significantly among the three groups (Table 2$)$, and $75 \%(98 / 130)$ of the subjects used an $\alpha_{1}$-blocker for $\geq 3$ month.

Frequency volume charts

Compared with the control group, those treated with imidafenacin showed a significantly greater reduction in the nocturnal micturition (Table 3). Daytime frequency improved in the IM twice/day group but not in the IM nightly group (Table 3 ). The nocturnal urine volume in the control group remained unchanged throughout the study period, but imidafenacin treatment tended to reduce nocturnal urine production; in particular, $0.1 \mathrm{mg}$ imidafenacin administered nightly reduced urine production significantly compared with that in the control group (Table 3). Further, in the IM nightly group, the 24-h urine volume remained unchanged, but NPi reduced significantly from the baseline. HUS and urine volume voided/void at night significantly improved in the IM twice/day group but not in the IM nightly group.

\section{N-QOL score, OABSS, IPSS and IPSS-QOL}

The total N-QOL score and subdomain scores at baseline were similar among the three treatment groups (Table 1). However, a marked increase was found in the N-QOL scores of the IM twice/day and IM nightly groups at 4 and 8 weeks (Table 3 ). The OABSS significantly improved in all groups from the baseline to 8-week values (changes from baseline: $-2.0 \pm 0.4$, control group; $-2.9 \pm 0.5$, IM twice/day group; $-2.2 \pm 0.3$, IM nightly group, not significant by Kruskal-Wallis test). Similarly, the IPSS also improved in all groups compared with the baseline values (changes from baseline: $-2.4 \pm 0.8$ in the control group, $-3.4 \pm 1.1$ in the IM twice/day group, and $-3.6 \pm 0.7$ in the IM nightly group, not significant by Kruskal-Wallis test). Further, the IPSS-QOL improved in all groups compared with the baseline values (changes from baseline: $-0.5 \pm 0.2$ in the control group; $-1.3 \pm 0.2$ in the IM twice/day group; and $-1.2 \pm 0.2$ in the IM nightly group, $p=0.013,<0.0001$ and $<0.0001$, respectively). Compared with the control group, the other two groups showed significantly improved IPSS-QOL scores $(p=0.0040$ and 0.0377 , respectively).

Tolerability and safety

Treatment-emergent AEs were reported by no subjects in the control group, $18.5 \%(10 / 54)$ of subjects in the IM twice/day group, and $25 \%(12 / 48)$ of subjects in the IM nightly group (Table 4 ). Imidafenacin plus $\alpha_{1}$-blocker treatment was not associated with an increased PVR (changes 


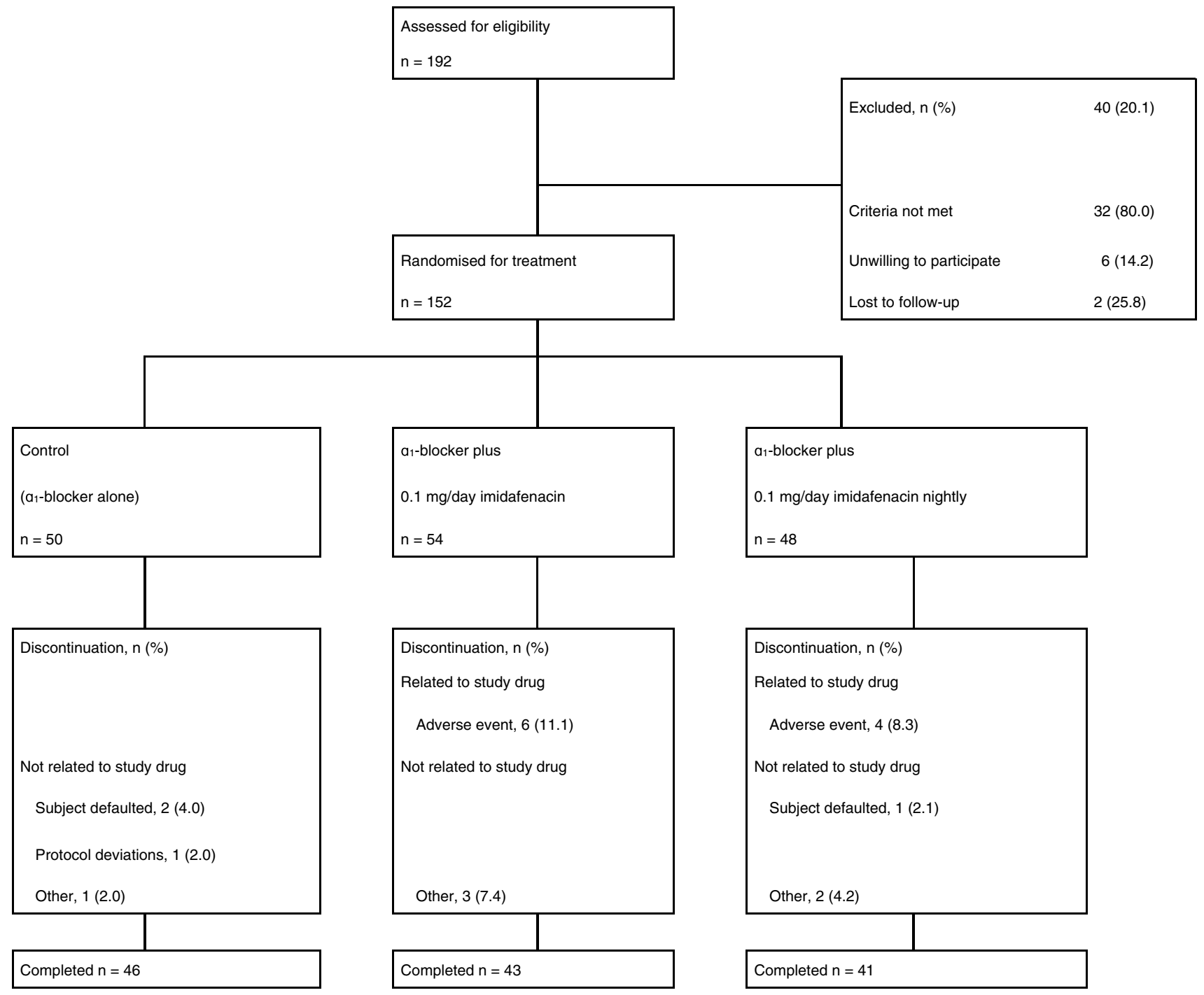

Fig. 1 Disposition of subjects assigned to the study treatments

from baseline: $3.9 \mathrm{~mL}$ in the control group, $6.8 \mathrm{~mL}$ in the IM twice/day group, and $7.5 \mathrm{~mL}$ in the IM nightly group). There were no reports of urinary retention.

\section{Discussion}

The Good-Night study is the first prospective randomised clinical trial that focused on nocturia, NP, and OAB symptoms, in order to evaluate the efficacy and safety of anticholinergics in male subjects on continued $\alpha_{1}$-blocker treatment. Additionally, this study showed the effect of anticholinergics on nocturia and NP in a real-life setting for the first time.

Nocturia has shown a poor clinical response to traditional OAB therapies, including anticholinergics [10]. Thus, other types of therapy will be needed to achieve a clinically significant reduction in nocturia. The use of the arginine-vasopressin analogue desmopressin in the treatment of NP has been studied in certain groups of patients, because it is an antidiuretic. Low doses of desmopressin administered to elderly subjects were found to reduce NP [21]. However, desmopressin is associated with the risk of hyponatremia, which is the main potentially serious associated $\mathrm{AE}$, and therefore, desmopressin is currently not recommended for patients $>65$ years old. Considering that the prevalence of nocturia and NP increases with age, the risk of AEs associated with some of the available medications warrants careful selection to optimise the therapeutic index.

A previous study on men with nocturia found an $83 \%$ incidence of NP; $20 \%$ of patients had NP alone, while $63 \%$ also had small nocturnal bladder capacity, bladder outlet obstruction, or sleep apnoea [22]. The current study seems to mirror real-life settings: $78 \%$ of the subjects had 
Table 1 Patient demographics and background

\begin{tabular}{lllll}
\hline & $\begin{array}{l}\text { Control } \\
(n=46)\end{array}$ & $\begin{array}{l}\text { IM twice/day } \\
(n=43)\end{array}$ & $\begin{array}{l}\text { IM nightly } \\
(n=41)\end{array}$ & $p$ value \\
\hline $\begin{array}{l}\text { Demographics } \\
\text { Age (years) }\end{array}$ & $73.3 \pm 6.7$ & $74.7 \pm 6.2$ & $74.7 \pm 7.6$ & $0.5376^{\dagger}$ \\
BMI (kg/m $\left.{ }^{2}\right)$ & $23.1 \pm 3.2$ & $23.1 \pm 4.0$ & $22.8 \pm 3.0$ & $0.8956^{\dagger}$ \\
$\begin{array}{l}\text { Prostate volume } \\
\quad(\mathrm{mL})\end{array}$ & $31.7 \pm 12.6$ & $28.1 \pm 11.3$ & $31.4 \pm 15.6$ & $0.4437^{\dagger}$ \\
PSA (ng/mL) & $2.6 \pm 2.2$ & $2.3 \pm 2.5$ & $2.4 \pm 2.0$ & $0.7707^{\dagger}$ \\
Comorbidity (\%) & & & & \\
Hypertension & 28.3 & 25.6 & 26.8 & $0.9689^{\ddagger}$ \\
Diabetes & 4.3 & 14.0 & 14.6 & $0.2070^{\ddagger}$ \\
Renal disease & 0.0 & 0.0 & 2.4 & $0.3154^{\ddagger}$ \\
Cerebrospinal & 6.5 & 7.0 & 12.2 & $0.6750^{\ddagger}$ \\
$\quad$ disease & & & & \\
PVR (mL) & $18.9 \pm 16.8$ & $15.9 \pm 13.5$ & $23.4 \pm 14.8$ & $0.7978^{\dagger}$ \\
OABSS & $7.84 \pm 2.3$ & $7.67 \pm 2.3$ & $7.79 \pm 2.4$ & $0.8612^{\dagger}$ \\
IPSS & $15.5 \pm 7.7$ & $14.6 \pm 7.5$ & $15.4 \pm 6.8$ & $0.7927^{\dagger}$ \\
IPSS-QOL & $4.24 \pm 1.04$ & $4.4 \pm 1.16$ & $4.25 \pm 1.24$ & $0.7915^{\dagger}$ \\
\hline
\end{tabular}

All values were expressed as mean $\pm \mathrm{SD}$

$B M I$ body mass index, $P S A$ prostate-specific antigen, $P V R$ post-void residual volume, OABSS Overactive Bladder Symptom Score, IPSS International Prostate Symptom Score, ANOVA analysis of variance

Control, $\alpha$-blocker alone; IM twice/day, $\alpha$-blocker plus $0.1 \mathrm{mg}$ imidafenacin twice daily; IM nightly, $\alpha$-blocker plus $0.1 \mathrm{mg}$ imidafenacin nightly

$\dagger p$ value: intergroup (control vs. $0.1 \mathrm{mg}$ imidafenacin twice/day or $0.1 \mathrm{mg}$ imidafenacin nightly); ANOVA

$\$ p$ value: intergroup (control vs. $0.1 \mathrm{mg}$ imidafenacin twice/day or $0.1 \mathrm{mg}$ imidafenacin nightly); Fisher's exact test

Table $2 \alpha$-Blocker use according to the enrolled subjects

\begin{tabular}{llccc}
\hline & $\begin{array}{l}\text { Control } \\
(n=46)\end{array}$ & $\begin{array}{l}\text { IM twice/day } \\
(n=43)\end{array}$ & $\begin{array}{l}\text { IM nightly } \\
(n=41)\end{array}$ & $p$ value $^{\dagger}$ \\
\hline$\alpha$-Blocker $[n(\%)]$ & & & \\
Tamsulosin & $13(28.3 \%)$ & $9(20.9 \%)$ & $15(36.6 \%)$ & 0.6373 \\
Naftopidil & $18(39.1 \%)$ & $19(44.2 \%)$ & $15(36.6 \%)$ & \\
Silodosin & $15(32.6 \%)$ & $15(34.9 \%)$ & $11(26.8 \%)$ & \\
Duration of $\alpha$-blocker use before study entry $[n(\%)]$ & \\
$\geq 1$ month & $6(13.0 \%)$ & $8(18.6 \%)$ & $7(17.1 \%)$ & 0.6887 \\
$\geq 2$ months & $4(8.7 \%)$ & $5(11.6 \%)$ & $2(4.9 \%)$ & \\
$\geq 3$ months & $6(13.0 \%)$ & $5(11.6 \%)$ & $2(4.9 \%)$ & \\
$\geq 6$ months & $30(65.2 \%)$ & $25(58.1 \%)$ & $30(73.2 \%)$ & \\
\hline
\end{tabular}

All values were expressed as mean $\pm \mathrm{SD}$

Control, $\alpha$-blocker alone; IM twice/day, $\alpha$-blocker plus $0.1 \mathrm{mg}$ imidafenacin twice daily; IM nightly, $\alpha$-blocker plus $0.1 \mathrm{mg}$ imidafenacin nightly

$\dagger p$ value: intergroup (control vs. $0.1 \mathrm{mg}$ imidafenacin twice/day or $0.1 \mathrm{mg}$ imidafenacin nightly); Fisher's exact test
OAB with NP. Further, the distribution of $\alpha_{1}$-blocker use in the treatment period, mean duration of $\alpha_{1}$-blocker use, and types of $\alpha_{1}$-blockers used before study entry did not differ significantly among the groups. Thus, the results of the current study were not influenced by the use of $\alpha_{1}$-blockers.

A well-known study known as the TIMES study showed the efficacy of combination therapy using $\alpha_{1}$-blockers and anticholinergics in male LUTS patients [13]. However, in TIMES study and other similar trials, the urine volume was not assessed. The objectives of the TIMES study thus differed from those of the Good-Night study, which focused on nocturia in male LUTS patients.

The primary endpoint in the Good-Night study, i.e. changes in night-time voiding frequency as determined using FVCs, was significantly reduced in the IM twice/day and IM nightly groups compared with that in the control group. This result is consistent with the findings of previous anticholinergic studies [13-15].

Unfortunately, in the present study, the baseline nocturia severity was lower in the control group than in the both study arms. Therefore, there may have been more room for improvement in the intervention groups than in the control group. However, no significant difference was found in the baseline OABSS among the groups, and OABSS nocturia score was similar among the groups (control group, $2.5 \pm 0.5$; IM twice/day, $2.7 \pm 0.5$; IM nightly group, 2.7. $\pm 0.5 ; p=0.2290$ by Kruskal-Wallis test, data not shown). Thus, it is unlikely that the differences in nocturia severity at baseline affected the results. In addition, the stratified analysis of patients with three or more nocturnal micturitions per night (baseline nocturnal micturition frequency: control group, $3.7 \pm 0.3$; IM twice/ day, $4.4 \pm 0.3$; IM nightly group, $3.9 \pm 0.2 ; p=0.1459$ by ANOVA) indicated that patients treated with imidafenacin showed a significantly greater reduction in the frequency of nocturnal micturition compared to the control group [mean changes \pm standard error (SE) from baseline to 8 weeks: control group, $-0.1 \pm 0.3$; IM twice/day: $-1.1 \pm 0.2$; IM nightly group, $-0.7 \pm 0.3, p=07671,<0.0001$ and 0.0238 , respectively].

The Good-Night study prospectively showed for the first time, to the best of our knowledge, that anticholinergics could reduce nocturnal urine volume in male LUTS patients with residual $\mathrm{OAB}$ symptoms, which is a key finding. Our data support those of previous reports: one previous study showed that imidafenacin decreased urine volume through suppression of C-fibres in the rat bladder [11], and another showed that it clinically reduced nocturnal urine volume [12], although the latter study was mainly conducted on women with incontinence and NP. Imidafenacin has a unique pharmacokinetics profile: its 


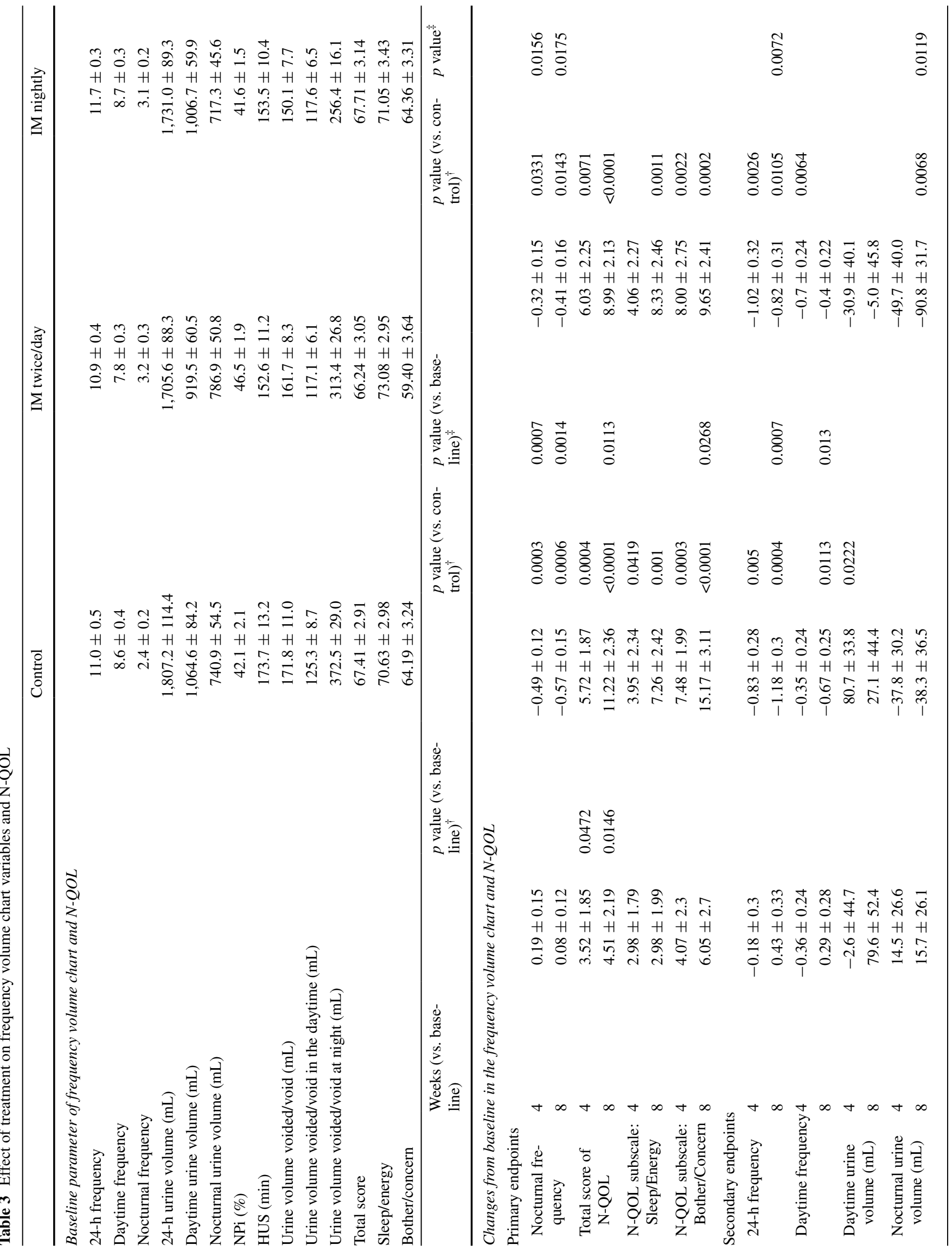




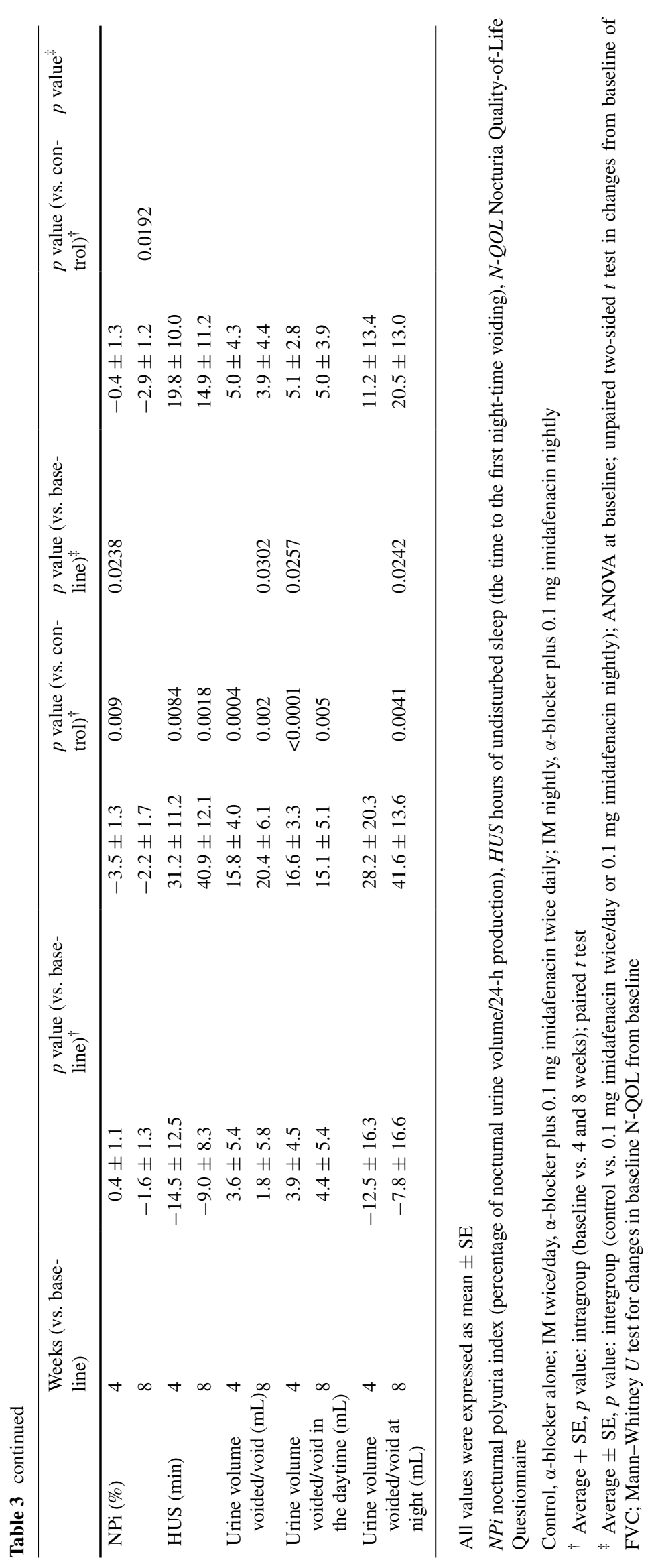


Table 4 Summary of treatment-emergent adverse events

\begin{tabular}{llll}
\hline & $\begin{array}{l}\text { Control } \\
(n=50)\end{array}$ & $\begin{array}{l}\text { IM twice/day } \\
(n=54)\end{array}$ & $\begin{array}{l}\text { IM nightly } \\
(n=48)\end{array}$ \\
\hline AEs $[n(\%)]$ & & & \\
Dry mouth & $0(0 \%)$ & $2(3.7 \%)$ & $6(12.5 \%)$ \\
Constipation & $0(0 \%)$ & $3(5.6 \%)$ & $1(2.1 \%)$ \\
Blurred vision & $0(0 \%)$ & $0(0 \%)$ & $1(2.1 \%)$ \\
Anorexia & $0(0 \%)$ & $1(1.9 \%)$ & $0(0 \%)$ \\
Insomnia & $0(0 \%)$ & $0(0 \%)$ & $1(2.1 \%)$ \\
Palpitations & $0(0 \%)$ & $1(1.9 \%)$ & $0(0 \%)$ \\
Oedema in the limbs & $0(0 \%)$ & $0(0 \%)$ & $1(2.1 \%)$ \\
Gastric ulcer & $0(0 \%)$ & $1(1.9 \%)$ & $0(0 \%)$ \\
Tremors & $0(0 \%)$ & $1(1.9 \%)$ & $0(0 \%)$ \\
Abdominal pain & $0(0 \%)$ & $1(1.9 \%)$ & $0(0 \%)$ \\
Pain & $0(0 \%)$ & $0(0 \%)$ & $1(2.1 \%)$ \\
Agnail & $0(0 \%)$ & $0(0 \%)$ & $1(2.1 \%)$ \\
Urinary AEs suggestive of & $0(0 \%)$ & $0(0 \%)$ & $0(0 \%)$ \\
$\quad$ AUR $[n(\%)]$ & & & \\
\hline
\end{tabular}

Control, $\alpha$-blocker alone; IM twice/day, $\alpha$-blocker plus $0.1 \mathrm{mg}$ imidafenacin twice daily; IM nightly, $\alpha$-blocker plus $0.1 \mathrm{mg}$ imidafenacin nightly; AE, adverse event; AUR, acute urinary retention

half-life in blood is $2.9 \mathrm{~h}$ (it is referred to as a short halflife anticholinergic), but its duration of receptor binding is longer in the bladder than in other organs $(6-9 \mathrm{~h}$ in the bladder, $1-3 \mathrm{~h}$ in the submaxillary gland; no observation in the brain), and its metabolite has no pharmacological activity [23, 24]. These properties differ from those of other anticholinergics. A possible explanation for how imidafenacin decreases the nocturnal urine volume is that it decreases the nocturnal urine volume directly by inhibiting bladder afferent nerves and/or indirectly by improving sleep disturbance. Elderly patients treated with sleeping pills have been shown to excrete smaller amounts of urine during the night [25], thus suggesting that sleep can result in reduced urine production.

In the current study, the IM twice/day group showed prolonged HUS, reduced night-time and daytime voiding frequency, and increased urine volume voided/void, as well as a tendency towards reduced NPi. These results are consistent with previous findings [12].

Anticholinergics are commonly believed to have no effect on urine production; however, the IM nightly group showed significantly reduced urine production compared with the control group. During the period when the blood level of imidafenacin was high, urination was suppressed, particularly in the night-time, when urine production increases in patients with NP. In contrast, when the blood level of imidafenacin decreased in the daytime, urination was no longer suppressed, so the fluid balance over $24 \mathrm{~h}$ was normalised. Therefore, anticholinergics with a long half-life may be poor agents for reducing urine production, because their antidiuretic effect persists throughout the day. For instance, while tolterodine has shown an antidiuretic effect in rats [11], the effect of extended-release tolterodine on urine production has not been clinically proven.

Another primary endpoint was the N-QOL score. Using this score as an efficacy index, the Good-Night study found that anticholinergic add-on therapy was more effective than $\alpha_{1}$-blocker monotherapy in male LUTS patients with residual OAB symptoms. It clearly showed that the N-QOL scores in the IM twice/day and IM nightly groups at 4 and 8 weeks were significantly increased from baseline and that the score in the IM twice/day group, but not the IM nightly group, was significantly higher than that in the control group. This improvement in HUS with imidafenacin may have important QOL and compliance benefits.

There have been concerns that the inhibitory effect of anticholinergics on detrusor muscle contraction could theoretically aggravate voiding difficulty, increase PVR, or cause urinary retention. However, in the Good-Night study, the PVR did not significantly increase from baseline to week 8 in any of the three groups.

The main study limitation is that this is a randomised but open-label, no-placebo and non-double-blinded study. To confirm the efficacy of a particular drug, a study should determine whether it is superior to a placebo and not inferior to a pre-existing drug. However, previous phase three trials' sub-analysis showed that imidafenacin $0.1 \mathrm{mg}$ twice/ day significantly reduced nocturnal frequency and nocturnal urine volume compared with placebo group [12]. Further research is required to draw a definitive conclusion regarding the reduction in nocturnal urine production by anticholinergics.

In conclusion, add-on therapy with a short half-life anticholinergic provides additional benefits for men with LUTS and nocturia who are undergoing $\alpha_{1}$-blocker therapy. Further, a short half-life anticholinergic may reduce nocturnal urine volume in addition to nocturnal frequency.

Acknowledgments This study was supported by the Supporting Center for Clinical Research and Education (SCCRE). The authors thank Dr. Urashima Mitsuyoshi at the Division of Molecular Epidemiology, Jikei University School of Medicine (Tokyo), for help with the random assignment, and the Osaka-Hokuriku male LUTS research study group for contributions to the success of this trial. We would like to express our sincere thanks to the following for their participation: Masato Kuribayashi and Takashi Shima, Kanazawa University Graduate School of Medical Science (Kanazawa); Masayuki Nakagawa, Kansai Medical University (Osaka); Hideo Tahara, Kinki University School of Medicine (Osaka); Katsuyuki Kuratsukuri, Osaka City University (Osaka); Yuto Nomi and Nobutaka Ubai, Osaka Medical College (Osaka); Yasushi Miyagawa and Tetsuya Takao, Osaka University Graduate School of Medicine (Osaka); Yoshitaka Aoki, Hideaki Ito, University of Fukui (Fukui); University of Toyama (Toyama); Fujita Memorial Hospital (Fukui); Fukui Social Insurance Hospital (Fukui); Hasegawa Hospital (Toyama); Hayashi Hospital 
(Fukui); Jujinkai Kimura Hospital (Fukui); Kimura Hospital (Fukui); Mimihara General Hospital (Osaka); and Nanto Municipal Hospital (Toyama).

Conflict of interest Osamu Yokoyama, first author, is a consultant for Astellas Pharma Inc. Pfizer Inc. Ono Pharmaceutical Co, Ltd., Nippon Shinyaku Co, Ltd., and Asahi Kasei Pharma Corp. All the other authors declare that they have no conflict of interest.

Ethical standard Performed in accordance with the institutional ethical guidelines ethical standards laid down in the 1964 Declaration of Helsinki its later amendments.

Open Access This article is distributed under the terms of the Creative Commons Attribution License which permits any use, distribution, and reproduction in any medium, provided the original author(s) and the source are credited.

\section{References}

1. van Kerrebroeck P, Abrams P, Chaikin D et al (2002) The standardization of terminology in nocturia: report from the Standardization Sub-committee of the International Continence Society. Neurourol Urodyn 21:179-183

2. Homma Y, Yamaguchi O, Hayashi K (2006) Neurogenic Bladder Society Committee. Epidemiologic survey of lower urinary tract symptoms in Japan. Urology 68:560-564

3. Coyne KS, Zhou Z, Bhattacharyya SK, Thompson CL, Dhawan $R$, Versi E (2003) The prevalence of nocturia and its effect on health-related quality of life and sleep in a community sample in the USA. BJU Int 92:948-954

4. Appell RA, Sand PK (2008) Nocturia: etiology, diagnosis, and treatment. Neurourol Urodyn 27:34-39

5. Asplund R (2004) Nocturia, nocturnal polyuria, and sleep quality in the elderly. J Psychosom Res 56:517-525

6. Kupelian V, Wei JT, O'leary MP, Norgaard JP, Rosen RC, McKinlay JB (2012) Nocturia and quality of life: results from the Boston area community health survey. Eur Urol 61:78-84

7. Weiss J, Blaivas JG (2000) Nocturia. J Urol 163:5-12

8. Stoevelaar HJ, Van De Beek C, Casparie AF, McDonnell J, Nijs HG (1999) Treatment choice for benign prostatic hyperplasia: A matter of urologic preference? J Urol 161:133-138

9. Smith AL, Wein AJ (2011) Outcomes of pharmacological management of nocturia with non-antidiuretic agents: Does statistically significant equal clinically significant? BJU Int 107:1550-1554

10. Brubaker L, FitzGerald MP (2007) Nocturnal polyuria and nocturia relief in patients treated with solifenacin for overactive bladder symptoms. Int Urogynecol J Pelvic Floor Dysfunct 18:737-741
11. Watanabe N, Akino H, Kurokawa T et al (2013) Antidiuretic effect of antimuscarinic agents in rat model depends on C-fibre afferent nerves in the bladder. BJU Int 112:131-136

12. Yokoyama O, Homma Y, Yamaguchi O (2013) Imidafenacin, an antimuscarinic agent, improves nocturia and reduces nocturnal urine volume. Urology 82:515-520

13. Kaplan SA, Roehrborn CG, Rovner ES, Carlsson M, Bavendam T, Guan Z (2006) Tolterodine and tamsulosin for treatment of men with lower urinary tract symptoms and overactive bladder: a randomized controlled trial. JAMA 296:2319-2328

14. Nishizawa O, Yamaguchi O, Takeda M, Yokoyama O, For the TAABO Study Group (2011) Randomized controlled trial to treat benign prostatic hyperplasia with overactive bladder using an $\alpha_{1}$ blocker combined with anticholinergics. LUTS 3:29-35

15. Yamaguchi O, Kakizaki H, Homma Y et al (2011) Solifenacin as add-on therapy for overactive bladder symptoms in men treated for lower urinary tract symptoms-ASSIST, randomized controlled study. Urology 78:126-133

16. Cornu JN, Abrams P, Chapple CR et al (2012) A contemporary assessment of nocturia: definition, epidemiology, pathophysiology, and management-a systematic review and meta-analysis. Eur Urol 62:877-890

17. Homma Y, Yamaguchi O, Imidafenacin Study Group (2009) A randomized, double-blind, placebo- and propiverine-controlled trial of the novel antimuscarinic agent imidafenacin in Japanese patients with overactive bladder. Int J Urol 16:499-506

18. Maggiore UL, Scala C, Venturini PL, Ferrero S (2013) Imidafenacin for the treatment of overactive bladder. Expert Opin Pharmacother 14:1383-1397

19. Homma Y, Kakizaki H, Yamaguchi O et al (2011) Assessment of overactive bladder symptoms: comparison of 3-day bladder diary and the overactive bladder symptoms score. Urology 77:60-64

20. Abraham L, Hareendran A, Mills IW et al (2004) Development and validation of a quality-of-life measure for men with nocturia. Urology 63:481-486

21. Asplund R, Sundverg B, Bengtsson P (1999) Oral desmopressin for nocturnal polyuria in elderly subjects: a double-blind, placebo-controlled randomized exploratory study. BJU Int 83:591-595

22. Chang SC, Lin AT, Chen KK, Chang LS (2006) Multifactorial nature of male nocturia. Urology 67:541-544

23. Yamada S, Seki M, Ogoda M, Fukata A, Nakamura M, Ito Y (2011) Selective binding of bladder muscarinic receptors in relation to the pharmacokinetics of a novel antimuscarinic agent, imidafenacin, to treat overactive bladder. J Pharmacol Exp Ther 336:365-371

24. Takeuchi T, Zaitsu M, Mikami K (2013) Experience with imidafenacin in the management of overactive bladder disorder. Ther Adv Urol 5:43-58

25. Yokoyama O, Matsuta Y, Yanai-Inamura H et al (2010) Zolpidem increases bladder capacity and decreases urine excretion in rats. Neurourol Urodyn 29:587-591 